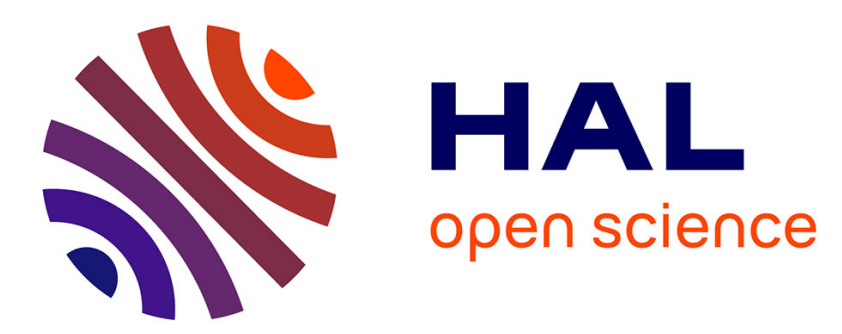

\title{
Study on the possible detection of Gamma Ray Bursts with the ANTARES neutrino telescope
}

\author{
G. Lelaizant
}

\section{To cite this version:}

G. Lelaizant. Study on the possible detection of Gamma Ray Bursts with the ANTARES neutrino telescope. THE MULTI-MESSENGER APPROACH TO HIGH-ENERGY GAMMA-RAY SOURCES, Jul 2006, Barcelone, Spain. 309, pp.441-445, 2007, 10.1007/s10509-007-9437-4 . in2p3-00186455

\section{HAL Id: in2p3-00186455 https://hal.in2p3.fr/in2p3-00186455}

Submitted on 9 Nov 2007

HAL is a multi-disciplinary open access archive for the deposit and dissemination of scientific research documents, whether they are published or not. The documents may come from teaching and research institutions in France or abroad, or from public or private research centers.
L'archive ouverte pluridisciplinaire $\mathbf{H A L}$, est destinée au dépôt et à la diffusion de documents scientifiques de niveau recherche, publiés ou non, émanant des établissements d'enseignement et de recherche français ou étrangers, des laboratoires publics ou privés. 


\section{Study on the possible detection of Gamma Ray Bursts with the ANTARES neutrino telescope}

Received: 10-27-06 / Accepted:

\begin{abstract}
The ANTARES telescope, currently in construction, is aiming to detect high energy neutrinos. Data from the first line of the detector, which became operational recently, demonstrates that the nominal time and space resolutions are achieved. Various models predict the emission of high energy neutrinos from astrophysical sources such as Supernova Remnants, Microquasars, Active Galactic Nuclei and Gamma Ray Bursts. With the custom designed data acquisition system of this detector, in combination with the existing satellite alert systems, the ANTARES telescope has an increased sensitivity for neutrinos from Gamma Ray Bursts compared to conventional time independent sources.
\end{abstract}

Keywords ANTARES telescope - neutrinos - Gamma Ray Bursts

\section{Introduction}

The ANTARES Collaboration is building a neutrino telescope at a depth of $2500 \mathrm{~m}$ in the Mediterranean Sea offshore from Toulon (France), with the main purpose of looking for high energy neutrinos from astrophysical sources [1]. The detector is a $3 \mathrm{D}$ array of photomultiplier tubes (PMTs) aiming to capture Cherenkov photons emitted by the passage of relativistic charged particles in seawater and especially from upward-going muons induced by neutrino interactions in the matter surrounding the detector. As neutrinos are neither deviated nor absorbed in the Universe thanks to their absence of electric charge and to their weak interaction with matter, the neutrino source direction can be determined. Reconstruction of muons trajectories is performed from the

\section{G. Lelaizant}

Centre de Physique des Particules de Marseille (CPPM)

Case 902 - 163, Avenue de Luminy 13288 Marseille Cedex 9, France

Tel.: +33491827647

Fax: +33491827299

E-mail: lelaizan@cppm.in2p3.fr measurements of time and position of PMT hits. Gamma Ray Bursts are particularly interesting because of the presumed neutrino emission in coincidence with the short burst of gamma-rays.

\section{The ANTARES telescope}

The ANTARES detector will consist of 12 mooring lines hosting a total of 900 Optical Modules (OMs), each with a $10^{\prime \prime}$ hemispherical PMT enclosed in a pressure resistant glass sphere. Each line, which is anchored on the seabed and has a height of about 450 metres, consists of five sectors of five storeys, each storey including three OMs, looking at $45^{\circ}$ downwards. Two storeys are separated by an electro-optical cable of about 15 metres. Two neighbouring lines are separated by $60 \mathrm{~m}$. Each line is connected to a junction box, which provides power and allows to send data via optical fibres to a shore station, located at 40 kilometres from the ANTARES site. The first line of the detector has already been deployed and is in operation since March 2006, while the complete 12-lines detector will be operational by the end of 2007 .

The ANTARES angular resolution is expected to be better than $0.3^{\circ}$ above a few $\mathrm{TeV}$. This requires a time calibration as well as a position reconstruction better than $0.5 \mathrm{~ns}$ and $10 \mathrm{~cm}$ respectively.

Some results obtained with the first line are described in the next section.

\section{Results from the first ANTARES line}

A complete line (Line 1) was deployed in February and connected in March 2006. The first data recorded in Spring 2006 have enabled to measure timing and position resolutions as well as to reconstruct the first atmospheric muons. 


\subsection{Time resolution}

The time resolution of the detector is monitored by LED Beacons, devices equipped with 36 synchronised LEDs, which can emit intense flashes of blue light in the sea water. Every line of the ANTARES detector is equipped with four LED Beacons, placed at various altitudes. Currently, an additional LED Beacon, located on an instrumented line, the Mini Instrumentation Line with Optical Modules (MILOM) [2], anchored at a distance of 80 metres from Line 1, is available. The timing resolution of the Line 1 PMTs are monitored by looking at the time dispersion of the detected LED Beacon photons with respect to the time of the flash. Figure 1 shows an example of the timing resolution obtained for one of the PMTs. The bottom Figure represents the time dispersion between the LED Beacon and a PMT located in the same horizontal plane at a distance of about 80 metres whereas the top one corresponds to a PMT at a distance from the LED Beacon of about 150 metres. Signal intensity loss as well as light scattering become important at this distance. All timing measurements are in good agreement with expectations.

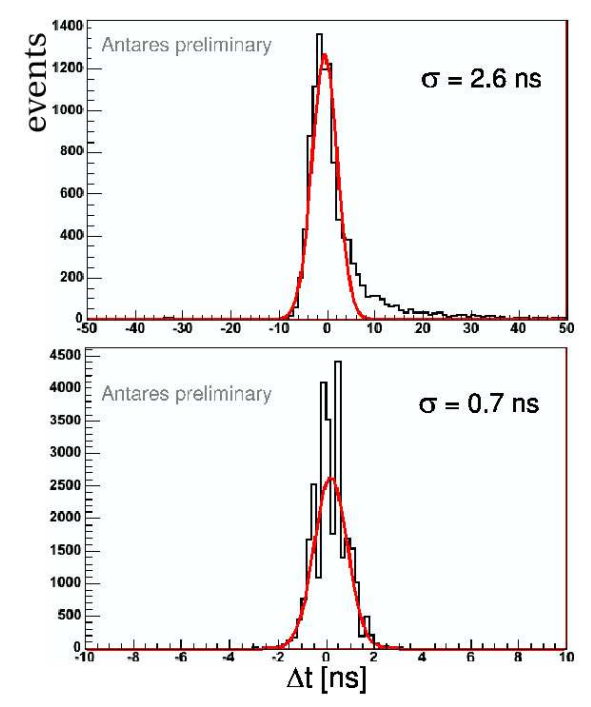

Fig. 1 Timing resolution measured by the LED Beacon system, which emits blue light flashes respectively detected on a $\mathrm{PMT}$ at a distance of $150 \mathrm{~m}$ (top) and $80 \mathrm{~m}$ (bottom) from the beacon

\subsection{Position resolution}

The geometry reconstruction of the detector is based on real time measurements of tiltmeters and compasses, placed in every storey, together with information obtained by a long baseline acoustic positioning system. This system is composed of five hydrophones for each line (one hydrophone for each sector) plus one transducer fixed at the bottom of each line and four transponders located on the sea floor, exchanging acoustic signals between each other. Knowing the sound velocity in the sea water, this system enables to triangulate hydrophone positions from acoustic travel time measurements with a precision of about $10 \mathrm{~cm}$.

Using the acoustic positioning system, we can monitor the displacements of the Line 1 hydrophones in order to reconstruct the line shape. As an example, Figures 2 and 3 show the displacement of the lowest one, placed on the bottom storey, during a period of two weeks. Figure 2 represents the horizontal displacement as function of time whereas Figure 3 shows the trajectory in the horizontal plane compared to the line anchor axis position. Figure 2 demonstrates a spatial resolution better than 10 $\mathrm{cm}$ as visible from the small dispersion of measurements taken in a short period.

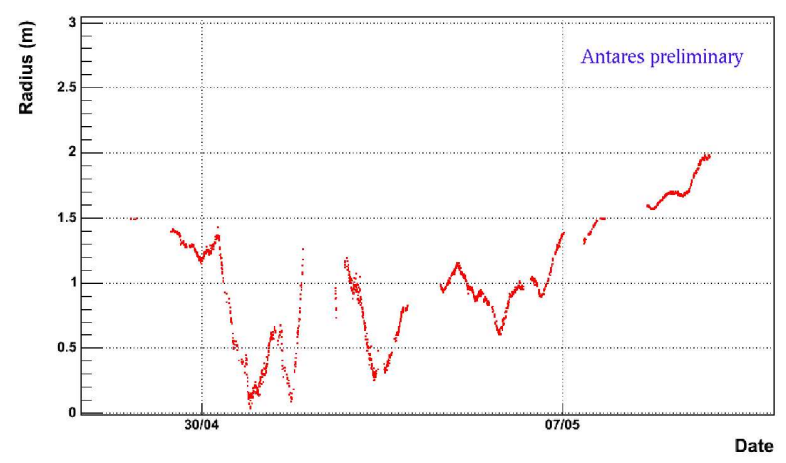

Fig. 2 Horizontal displacement of the lowest hydrophone as a function of time during a two weeks period

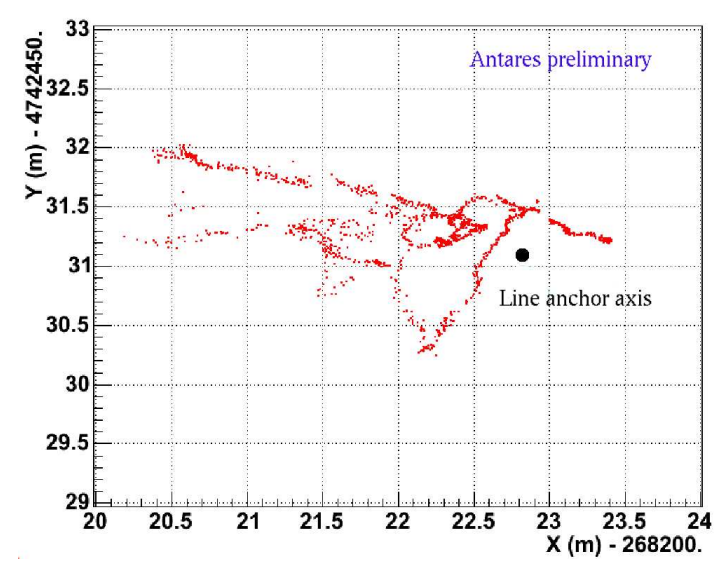

Fig. 3 Trajectory of the lowest hydrophone during 2 weeks. The coordinates are given in the UTM geodesic system. 


\subsection{Reconstruction of atmospheric muons}

Although the OMs are pointing downwards, the ANTARES detector has a significant sensitivity to downwardgoing atmospheric muons. The high bandwidth of the detector link to shore allows to send all the hits to the shore station where they are processed by a dedicated computer farm running an online data filter, implemented in software. The data filter algorithm looks for a set of PMT hits compatible with a muon track passing through the detector. First, it uses a reduced data set of local coincidences. A local coincidence is defined by a pair of hits occuring in a 20 ns time window on two PMTs of the same storey. Then, the algorithm searches for correlations between these hits according to a causality spacetime window which links the time difference between two hits and the distance between the positions of these two hits. Typically a minimum of 5 local coincidences is required during a $4 \mu s$ time window in order to define a physics event. If this trigger condition is met, all hits within this time window are recorded for offline analysis. For Line 1, the reconstruction of the muon track is performed offline by a $\chi^{2}$ minimisation of the time and altitude of all the hits of the physics event in order to determine the zenith angle of the track as well as its position. An example of such a muon reconstruction is shown on figure 4 .

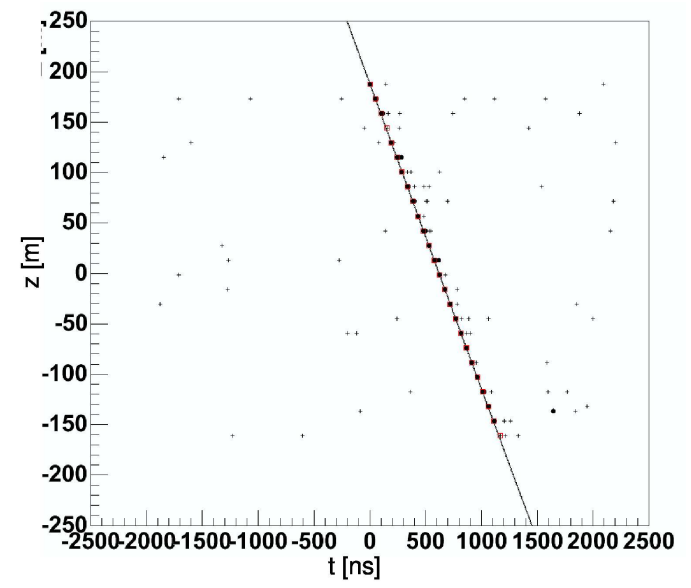

Fig. 4 Hit altitude (relative to mid detector) of a reconstructed atmospheric muon track as a function of hit time

\section{Gamma Ray Bursts: a potential source for the ANTARES telescope}

A standard reconstruction method has been implemented for atmospheric or cosmic muon neutrinos. The data taking efficiency is however limited by the need to remove the background. For Gamma Ray Bursts (GRBs), a special data taking, data filtering and reconstruction have been studied [3], [4] that show an increased sensitivity to neutrinos during a GRB.

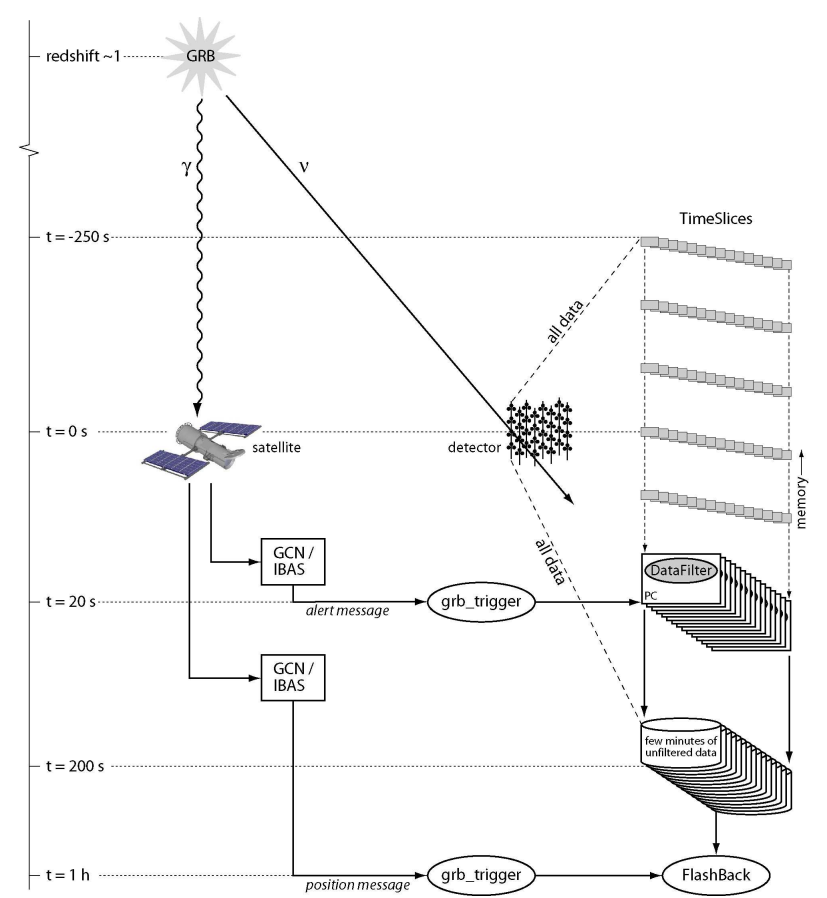

Fig. 5 Special data taking and data filtering for GRB neutrino detection

GRBs are short and intense flashes of $\mathrm{MeV}$ gamma rays at various locations in the sky and at random times. The total energy emitted in gamma rays ranges from $10^{51}$ erg to $10^{54} \mathrm{erg}$. In most models, to explain the emission of high energy photons in a specific direction, matter is ejected at relativistic speeds during the burst leading to the acceleration of electrons and baryonic matter to high energies in a jet. Accelerated protons can then interact with photons, already present in the medium or emitted by synchrotron radiation, and produce neutrinos via pion production and decay. It is believed that internal shocks, between different shells propagating at different speeds (in the most widely accepted model : the fireball model [5]), explain the prompt emission of gamma rays whereas an emission of radiation at other wavelengths, the afterglow, which follows the prompt emission, originates from the interaction with the interstellar medium. The neutrinos which are produced in these two processes have energy ranges of $100 \mathrm{TeV}$ and $100 \mathrm{PeV}$ respectively [6]. The duration of the prompt emission of the gamma rays ranges from $30 \mathrm{~ms}$ to $1000 \mathrm{~s}$. Neutrino emissions are expected to be simultaneous or sometimes earlier (10 $100 \mathrm{~s}$ before) than gamma rays. The time scales related to GRB neutrinos justify the special data taking mode of ANTARES, which allows to read out an unfiltered data sample covering a period which extends from $250 \mathrm{~s}$ before the alert to a few minutes after. 
GRB alert systems are used to trigger the special data taking mode. There are two such alert systems: the GRB Coordinates Network (GCN), initially named BATSE Coordinates Distribution Network, and the INTEGRAL Burst Alert System (IBAS). Satellites that detect GRBs, like Swift and INTEGRAL, are connected to these systems. Both systems send an alert message when a GRB is detected by one of these satellites. The delay between the $\gamma$-ray detection and the alarm broadcast by GCN or IBAS is less than $100 \mathrm{~s}$ for most GRBs.

The special data taking mode of ANTARES, which is sketched on Figure 5, starts after the reception of the GRB alert message. All data are written to disk during a period of three minutes. In addition, the data in the memories of the data processing $\mathrm{PCs}$ are written to disk, which correspond to the data that were recorded before the alert message arrived and possibly before the $\gamma$-rays were detected by the satellite. As a consequence, all raw data that were recorded before, during and after the GRB (no data loss) are stored on disk, allowing a dedicated offline analysis on a full data sample which covers a period of a few minutes (i.e. a few times the burst duration). This data taking mode is only possible for short events because of the high background, and cannot be applied for continuous sources.

Satellite warning systems enable to confirm the alert and also to determine the position of the gamma source after a delay varying from one to six hours for most of the GRBs. Knowing the source position, a special data filter (FlashBack algorithm) looks offline for muon tracks from the given GRB direction within the unfiltered data set. Then a special reconstruction method is applied on these data. A likelihood ratio method is used to quantify the compatibility of each event with a muon coming from the direction of the GRB. This procedure leads to an extra sensitivity to signal from the GRB.

As shown in Figure 6, this procedure i.e. an offline analysis of an unfiltered data set knowing the signal direction and the GRB time, increases the sensitivity to GRB neutrinos compared to the standard ANTARES analysis method applied to continuous sources. Studies on Monte Carlo simulations for different GRBs occurring at various zenith angles were performed. The corresponding neutrino effective areas obtained with the GRB analysis method are shown on Figure 7. The same analysis method can be used for continuous sources with known position on the sky, however, for these sources the detection efficiency will not increase as much as for GRBs because of the GRB transient feature.

\section{Conclusion}

The ANTARES Collaboration has deployed the first full line of its neutrino telescope, which takes data since March 2006. Data of this first line taken during Spring 2006 show that the detector specifications are satisfied.

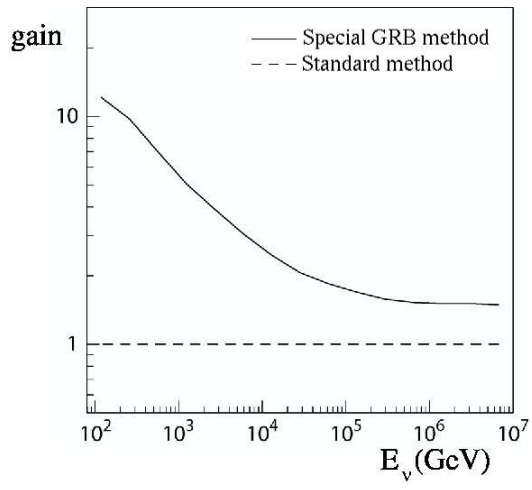

Fig. 6 Gain in sensitivity for GRB neutrinos using the special GRB method compared to the standard analysis method

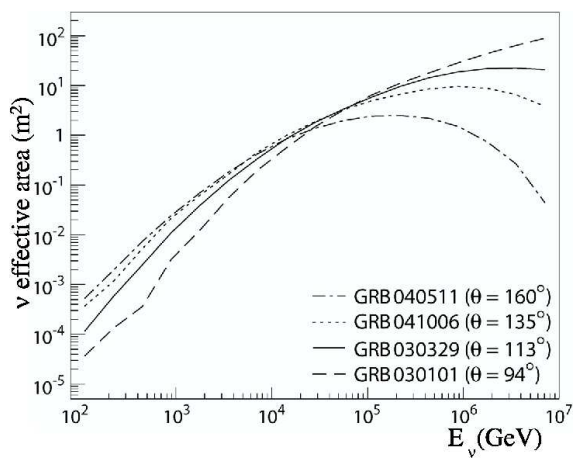

Fig. 7 Effective areas for neutrinos before traversing the Earth and interacting for GRBs at different zenith angles using the special GRB method

In particular, they validate the time and spatial resolutions. The standard filtering and reconstruction method, developed for muon neutrino astronomy, has already enabled the reconstruction of downgoing atmospheric muons. A specific way of data taking, filtering and analysis, in combination with existing satellite alert systems data, should enable to study the existence of space-time correlations between high energy neutrinos and Gamma Ray Bursts. The experience with the first complete ANTARES line gives confidence for the operation of the next lines which are scheduled to be deployed during 2006 and 2007.

\section{References}

1. E. Aslanides et al. ANTARES Collaboration, the ANTARES Proposal (astro-ph/9907432)

2. The ANTARES Collaboration: astro-ph/0606229, to be published in Astroparticle Physics

3. M.C. Bouwhuis: Detection of Neutrinos from Gamma Ray Bursts, Ph.D. thesis (2005)

4. M.C. Bouwhuis (on behalf of the ANTARES Collaboration), Nuovo Cimento C 28 (2005) 805-808

5. T. Piran: Gamma-Ray Bursts and the Fireball Model, Phys. Rep. 314(1999) 575

6. E. Waxman, J. Bahcall: High energy neutrinos from astrophysical sources: an upper bound, Phys. Rev. Lett. D59 (1999) 23002 